\title{
Herbal therapy for men with erectile dysfunction
}

\author{
Bichitra N Nayak PhD ${ }^{1}$, Harpal S Buttar DVM PhD FICN²
}

BN Nayak, HS Buttar. Herbal therapy for men with erectile dysfunction. Curr Res Cardiol 2015;2(1):30-34.

Erectile dysfunction (ED) or male impotence is defined as inability of a man to achieve or maintain penile erection sufficient for sexual activity. It is primarily a neuronal and endothelial dysfunction of the corpus cavernosum of penile tissue, and is partly characterized by reduced production of nitric oxide (NO). Other factors that may contribute to the pathogenesis of ED include androgen deficiency in aging men, hypertension, high cholesterol levels, atherosclerosis, diabetes mellitus, diseases of the prostate and heart, and anatomical deformity of the penis. ED may also be caused by some medications, prostate surgery and spinal cord injury. Psychological and social conditions such as stress, depression and unhappy marital relationship may contribute to the problem. Chronic infections and inflammation can also contribute to the disease process.

G enerally, erectile dysfunction (ED) is a neurovascular condition directly involving the endothelium of the corpora cavernosal arterial blood vessels in the penis, and is indirectly linked to cardiovascular diseases. The underlying mechanisms of ED are, however, complex and involve psychogenic, neurogenic, hormonal and vascular factors. ED occurs in aging men, with a prevalence of $52 \%$ in men 40 to 70 years of age (1-3). Conditions that may cause ED include hypertension, diabetes, diseases of the prostate and heart, and obesity. ED may also be caused by the effects of certain medications as well as physical injury or anatomical deformity of the penis (4), or may result from endocrine disorders such as low testosterone, hypogonadism, adrenal insufficiency and hypothyroidism. Changes in blood flow to the male reproductive organs as a result of hardening of the arteries or atherosclerosis, hypertension and hypercholesterolemia may result in ED. It is generally accepted that there are vascular and neuropathic components to the pathophysiology of the disease, and ED has been recognized as a potential indicator of underlying cardiovascular disease. Chronic infections and/or inflammation of the prostate and irritation of the bladder may contribute to the pathogenesis of ED.

In diabetes, vasoconstrictors and vasoactive factors are increased in addition to structural changes and attenuation of relaxation responses in the corpus cavernosum. A shifting of the balance of vasoactive factors occurs such that relaxation factors (eg, nitric oxide [NO]) are inhibited and contractile factors are induced in microvascular disease. With epidemiological predictions suggesting that the incidence of diabetes mellitus will increase to 300 million by 2025, management of diabetes-induced ED is increasingly important.

\begin{abstract}
ED is linked to an increased risk for cardiovascular diseases and stroke. Several orally active drugs (sildenafil, vardenafil, tadalafil, avanafil) are currently prescribed for treating ED to improve the arterial blood flow to the penile tissue. Medicinal plants and their extracts have been used in traditional medicine in southwest Asia and other countries to treat ED. The current review focuses on four medicinal plants that have been used as aphrodisiacs for enhancing sexual performance and for the treatment of ED. These plants include Eurycoma longifolia Jack (tongkat ali); Chlorophytum borivilianum (safed musli); Withania somnifera (ashwagandha); and Pausinystalia johimbe (formerly known as Corynanthe johimbe). Suggested mechanisms of action for each of the plant extracts will be discussed.
\end{abstract}

Key Words: Endothelial dysfunction; Erectile dysfunction; Herbal therapy for erectile dysfunction; Impotence in men

ED may also be psychogenic in origin and the treatment may involve resolution of psychoemotional problems that frequently occur in the dyadic relationship, regardless of the etiology of the ED. Psychological conditions and social problems such as stress, depression and unhappy relationships may contribute to the problem.

ED may occur at any age, but tends to have a greater psychological effect when it occurs in midlife. ED invokes stress related to midlife intimacy and the physiological realities of aging. Although the prevalence of ED increases with age, it is not an inevitable consequence of aging. Rather, ED becomes more prevalent as men grow older because of its relationship with various age-related diseases. Several studies have found that age is an independent risk factor for severe ED, even after adjustment for other age-related diseases. The aging male requires more penile stimulation; it takes longer to get an erection and the erection may not be hard enough for vaginal penetration. Also, it takes more time to reach ejaculation in elderly individuals. Absence of sexual interest in the partners of older men can lead to ED simply by the man not receiving sufficient direct penile stimulation. Testosterone replacement therapy for aging men has become a topic for discussion among health care providers. There are no established norms for testosterone levels in aging men. Studies in healthy men show that testosterone levels, particularly free bioactive testosterone levels, decline with age although there is considerable interindividual variation. The percentage of men who actually become 'testosterone deficient' is unknown. The diagnosis of androgen deficiency in aging men is associated with a wider range of symptoms than a mere impact on hormone levels per se. If the patient has no clinical signs of an androgen deficiency, testosterone replacement therapy will have no clinical effect.

${ }^{1}$ Richardson Centre for Functional Foods and Nutraceuticals, University of Manitoba, Winnipeg, Manitoba; ${ }^{2}$ Department of Pathology $\mathcal{E}$ Laboratory Medicine, Faculty of Medicine, University of Ottawa, Ontario

Correspondence: Dr Harpal S Buttar, Department of Pathology $\mathcal{E}$ Laboratory Medicine, Faculty of Medicine, University of Ottawa, 451 Smyth Road, Ottawa, Ontario K1H 8M5. Telephone 613-824-1532,e-mail hsbuttar@bell.net 


\section{PATHOPHYSIOLOGY OF ED AND PRIAPISM}

The neurovascular mechanism of erection is complex and involves multiple factors including hormones, neurotransmitters, elements of the autonomic nervous system (sympathetic and parasympathetic) and vasodilators such as NO. The common causes of ED include psychogenic disturbance with failure to relax cavernous smooth muscle, arterial insufficiency as a result of atheromatous disease, damage to the parasympathetic nervous system, spinal cord injury, diabetes or following pelvic surgery such as radical prostatectomy, radical cystectomy or bowel resection (4). It is important to note that cavernous nerves are unique in that although they belong to the autonomic nerves system they do not release either acetylcholine (Ach) or norepinephrine; however, they release $\mathrm{NO}$ in the penis. $\mathrm{NO}$ relaxes the smooth muscle of the corpora cavernosa via cyclic GMP (cGMP), allowing expansion of the cavernosal lacunar spaces, blood flow and erection. Thus, $\mathrm{NO}$ is not a direct dilator of the smooth muscle of cavernosal bodies, but it is an important mediator in this process. Erectile function may also be adversely affected by cigarette smoking, excess alcohol consumption, obesity and systemic diseases such as mononucleosis, hepatitis, HIV and cancer. Some men are prone to develop an erection that fails to subside after ejaculation (ie, priapism). The condition is associated with sickle-cell disease and leukemia, or may be a result of intracavernosal injection of drugs such as prostaglandin $E_{1}$ (4). Peyronie's disease causes a physical bend in the erect penis and also contributes to ED.

\section{CURRENT THERAPY FOR ED}

Currently, there are four orally active drugs are available to treat ED. These include: sildenafil citrate (Viagra [Pfizer, USA]), vardenafil hydrochloride (Levitra [Bayer, Germany]), tadalafil (Cialis [Eli Lilly, USA]) and avanafil (Stendra, Spedra [Vivus Inc, USA]). These drugs inhibit the enzyme phosphodiesterase type 5 (PDE-5), which is responsible for the hydrolysis of cGMP. PDE-5 inhibitors and cGMP act as effectors of dilation of smooth muscle of cavernosal bodies. PDE-5 inhibitors are contraindicated in patients taking any kind of nitrate therapy for angina, and may not be appropriate for men with certain health conditions, such as severe heart disease, heart failure, history of stroke or heart attack, uncontrolled high blood pressure or diabetes, and patients with pigmental retinopathy. PDE-5 inhibitors are less effective in men with diabetes and men who have been treated for prostate cancer. PDE-5 inhibitors are also not effective in men with retinitis pigmentosa, a genetic disease involving PDE-5 deficiency. The common side effects of PDE-5 inhibitors include gastrointestinal upset, headache, nasal congestion, back pain and dizziness. The PDE-5 inhibitors may interact with other medications including antihypertension drugs. Nonetheless, the PDE-5 inhibitors are generally safe and effective for most men. The primary mechanism of action of these drugs is through the mediation of NO. NO is one of the key molecules involved in ED. It is a short-lived, highly permeable, pleiotropic, gaseous molecule, secreted from the postganglionic cavernosal parasympathetic nerves, endothelium of the cavernosal blood vessels, platelets in the cavernosal sinuses and phagocytic cells (monocytes, macrophages and neutrophils). NO acts on platelets to inhibit platelets adhesion and aggregation. NO causes relaxation of the smooth muscle of the cavernosal blood vessels of the penis, leading to vasodilation, tumescence and stimulation. Release of $\mathrm{NO}$ in the corpus cavernosum of the penis during stimulation activates the enzyme guanylate cyclase, which results in increased levels of cGMP, producing smooth muscle relaxation in the corpus cavernosum and resulting in increased blood flow (5). NO is mainly produced from cavernosal nerves, which are nonadrenergic, noncholinergic nerves within the penis, and acting via its second messenger cGMP. It has been suggested that maintaining normal body weight and mild exercise, as well as dietary supplementation of folic acid, zinc, calcium, vitamin $\mathrm{C}$, vitamin $\mathrm{E}$ and $\mathrm{L}$-arginine, a precursor of $\mathrm{NO}$, can support the biochemical pathway leading to
$\mathrm{NO}$ release (6). NO is an effector molecule that is involved in a number of intracellular functions such as vasorelaxation, endothelial regeneration, inhibition of leukocyte chemotaxis and platelet adhesion (7). A small proportion of autonomic nerves do not release either Ach or norepinephrine (8). For example, the cavernous nerves predominantly release $\mathrm{NO}$ in the penis. The exact mechanism is not known, but it is believed to be through increased intracellular calcium. Another gaseous molecule produced in the corpora cavernosa is hydrogen sulphide $\left(\mathrm{H}_{2} \mathrm{~S}\right)$, which is also known to be involved in erectile function (9). $\mathrm{H}_{2} \mathrm{~S}$ activates ATP-sensitive potassium channels in smooth muscle cells. Some reports indicate that $\mathrm{NO}$ acts in large vessels and $\mathrm{H}_{2} \mathrm{~S}$ in small vessels. A high level of tumour necrosis factor-alpha has been shown in ED patients (10). Although current ED therapies using PDE-5 inhibitors are safe and effective, approximately $40 \%$ of ED patients do not respond to currently available treatment $(11,12)$. For these patients, herbal therapy may be useful.

The basis of ED herbal therapies is that they are anti-inflammatory, antioxidant and immunomodulatory, and can stimulate testosterone production. On the other hand, the synthetic drugs act via NO. The principal mediator of the relaxation of corporal smooth muscle of the penis has been shown to be $\mathrm{NO}$, which is released mainly from parasympathetic nerves and endothelium (13). NO is believed to relax the corporal smooth muscle by activating soluble guanylate cyclase to increase cGMP content $(14,15)$. Penile rigidity depends on maximizing inflow of blood while minimizing outflow (6). The increased blood flow in the cavernous sinuses puts pressure on the walls of the surrounding veins, causing the lumen of the veins narrow, temporarily interfering with the flow of blood but causing tumescence. Normally, the parasympathetic nerve produces Ach. Ach acts on muscarinic receptors and nicotinic cholinergic receptors. When the parasympathetic nerve is stimulated, preganglionic neurons release Ach at the ganglion, which acts on nicotinic receptors on postganglionic receptors. Postganglionic neurons then release Ach to stimulate muscarinic receptors of the target organs. The muscarinic receptor M3, present in the endothelial cells and smooth muscle, is activated, and the M2 receptor in the heart may also be activated. This may result in the production of Ach, which can cause endothelial cells to produce NO. Ach released from postganglionic parasympathetic nerves acting through G-protein-mediated muscarinic receptors and nicotinic cholinergic receptors helps to release NO. Normally, M1, M2 and M3 receptors are found in secretory glands, heart, smooth muscle and endothelial cells, respectively. M1, $\mathrm{M} 2$ and $\mathrm{M} 3$ receptors cause activation of phospholipase $\mathrm{C}$ and generate inositol trisphosphate and diacylglycerol, which increase calcium. Activation of M4 may inhibit adenylate cyclase, decreasing the messenger cyclic AMP. This mechanism may be involved in the relaxation and contraction of cavernosal smooth muscle cells.

\section{CONTRAINDICATIONS OF PDE-5 INHIBITORS AND ADVERSE REACTIONS}

Sildenafil citrate, tadalafil and vardenafil hydrochloride are contraindicated in patients with a known hypersensitivity to any organic nitrates (oral, sublingual, transdermal or by inhalation) due to the risk of developing potentially life-threatening hypotension. Concomitant use of PDE-5 inhibitors is contraindicated with HIV protease inhihibitors (indinavir, ritinovir, saquinavir) as well as ketoconazole, itraconazole, cimetidine and erythromycin, because these drugs are potent inhibitors of cytochrome P450 3A4 and can raise the plasma levels of PDE-5 inhibitors. Coadministration of PDE-5 inhibitors to patients taking alpha-adrenergic blockers such as alfluzocin or tamsulosin may lead to symptomatic postural hypotension, including dizziness and lighheadedness in some individuals.

Several serious adverse effects have been reported following the intake of PDE-5 inhibitors. These include loss of vision in one or both 
eyes (ie, nonarteritic anterior ischemic optic neuropathy), intracerebral hemorrhage, and bilateral sensorineural hearing loss or deafness.

\section{HERBAL THERAPIES FOR ED}

Extracts from medicinal plants have been used for considerable period of time in many parts of the world, particularly in Southwest Asia, to treat ED (16). The current review focuses on four botanical medicinal plants, the roots of which are used in enhancing sexual performance and in the treatment of ED: Eurycoma longifolia Jack (tongkat ali); Chlorophytum borivilianum (safed musli); Withania somnifera (ashwagandha); and Pausinystalia johimbe (yohimbine, formerly known as Corynanthe johimbe).

\section{E longifolia Jack (tongkat ali)}

E longifolia is a medicinal plant (family Simaroubaceae) native to Indonesia, Malaysia, Thailand, Vietnam and Laos. The root extract has been found to be the most powerful herbal aphrodisiac (17). Tongkat ali extracts contain many alkaloids, quassinoids, phenolic compounds, tannins, high-molecular-weight glycoproteins and mucopolysaccharides. The main bioactive compounds are eurycomaoside, eurycolactone, eurycomalactone, eurycomanone and pasakbumin-B. It is considered to be natural 'Viagra'. It increases sexual desire, and enhances performance and general well-being (1719). In addition to its aphrodisiac effect, other medicinal effects, such as antimalarial, antibacterial, antipyretic, antiulcer and antitumour effects, have been reported $(20,21)$. Root decoction has been used as a general tonic $(18,22)$. Laboratory animal studies show that root extract enhances sexual characteristics and performance in rodents (22-25). In a study involving a boar model, it was found that E longifolia root extract-treated boars increased sperm counts and semen volume; the effect was attributed to increased level of plasma testosterone (26). Reports also suggest that E longifolia extract reverses the inhibitory effects of estrogen on testosterone production and spermatogenesis in rats (27). Oral administration of E longifolia extract to inexperienced castrated male rats produced dose-dependent increases in sexual performance (28). Zakaria et al (29) found that eurycomanone, a potential bioactive compound in the root extract of E longifolia, induced apoptosis in hepatocarcinoma (Hep G2) cells. Furthermore, their work suggested that eurycomanone was cytotoxic to Hep G2 cells and less toxic to normal Chang's liver and WLR-68 cells. Tambi and Imran (19) investigated the effects of water-soluble extract of the root of E longifolia Jack and found that the extract increased semen volume, sperm concentration, percent of normal sperm morphology and sperm motility in male partners of subfertile couples with idiopathic infertility. Supplementation with E longifolia elevated the testosterone levels and upregulated osteoprotegerin gene expression in male Sprague-Dawley rats (30).

\section{C borivilianum (safed musli)}

C borivilianum (family Liliaceae) is native to India. Analysis of C borivilianum root revealed a composition of $12 \%$ to $17 \%$ saponins, $1.9 \%$ to $3.5 \%$ stigmasterol, $0.79 \%$ arabinose, $3.8 \%$ galactose, $0.73 \%$ glucose and $0.78 \%$ rhamnose (31). For dried root powder, the recommended dose is $5 \mathrm{~g}$ and the extract dose is $500 \mathrm{mg}$. It is used as an aphrodisiac and to cure ED, improve semen quality and volume. It eliminates premature ejaculation, improves general well-being and vitality, and increases stamina and libido. Visavadiya and Narasimhacharya (31) have demonstrated that administration of $C$ borivilianum $(0.75 \mathrm{~g}$ and $1.5 \mathrm{~g}$ root powder per rat per day for four weeks) to hypercholesteremic rats significantly increased highdensity lipoprotein cholesterol levels and decreased plasma and hepatic lipid profiles. Furthermore, the treatments also resulted in increased excretion of fecal cholesterol, sterols and bile and increased superoxide dismutase levels. Kenjale et al (32) evaluated the aphrodisiac and spermatogenic potential of the aqueous extract of dried roots of $\mathrm{C}$ borivilianum in rats. $\mathrm{C}$ borivilianum was given orally at doses of $125 \mathrm{mg} / \mathrm{kg} /$ day and $250 \mathrm{mg} / \mathrm{kg} /$ day. Viagra $4 \mathrm{mg} / \mathrm{kg} /$ day (sildenafil citrate) was administered as a control. Sexual behaviour was monitored $3 \mathrm{~h}$ later using a receptive female. Their sexual behaviour was monitored on days $1,7,14,21$ and 28 of treatment by pairing with proestrous female rats. For sperm count, the treatment was continued further in all groups except for the Viagra group for 60 days. At $125 \mathrm{mg} / \mathrm{kg}$, C borivilianum had marked aphrodisiac action, as demonstrated by increased libido, sexual vigour and sexual arousal in the rats. Similarly, at the higher dose $(250 \mathrm{mg} / \mathrm{kg})$, all the parameters of sexual behaviour were enhanced, but showed saturation effect after 14 days. On day 60, the sperm count increased significantly in both the C borivilianum-treated groups $(125 \mathrm{mg} / \mathrm{kg} / \mathrm{day}$ and $250 \mathrm{mg} / \mathrm{kg} / \mathrm{day})$ in a dose-dependent manner. The administration of $\mathrm{C}$ borivilianum extract has been found to be useful for the treatment of premature ejaculation and oligospermia (32). Supplementation with C borivilianum root $250 \mathrm{mg} / \mathrm{kg} /$ day and $500 \mathrm{mg} / \mathrm{kg} /$ day to streptozoticin-induced diabetic male rats for 28 consecutive days improved sperm morphology, and reduced oxidative stress and formation of free radicals (33). In case of streptozotocin- and alloxan-induced hyperglycemia, the aqueus extracts from C borivilianum resulted in improved sexual performance compared with diabetic control $(34,35)$.

\section{W somnifera (ashwagandha)}

W somnifera (ashwagandha), also referred to as winter cherry (family Solanaceae), grows in Africa, the Mediterranean, India, Pakistan, Bangladesh, Afghanistan, South Africa, Egypt, Morocco, Congo and Jordan (36). The roots of the plant contain steroid alkaloids and steroidal lactone, which are the main constituents of ashwagandha; these compounds are referred to as withanolides. Among the various alkaloids, withnine is the main constituent. The other alkaloids are somniferin, somnine, somniferine, withananine, pseudowithanine, tropine, pseudotropine, cuscohygrine, anferine and anhydrine. Two acyl steryl glucosides (sitoindoside VII and sitoindoside VIII) have been isolated from the root. The withanolides contain a C28 steroidal nucleus with a C9 side chain and six-membered lactone rings. Ashwagandha root also contains flavonoids and many ingredients of the withanolide class. It has several medicinal applications (aphrodisiac, liver tonic, anti-inflammatory agent, astringent), and is used to treat bronchitis, asthma, ulcers, insomnia, senility and dementia. Clinical trials and studies involving animal models support the use of ashwagandha for anxiety, cognitive and neurological disorders, inflammation and Parkinson's disease. It also provides cryoprotective benefits to patients undergoing radiation and chemotherapy, and shows beneficial effects for nervous exhaustion. W somnifera is used as an aphrodisiac, sedative and rejuvenative, and is also used to treat chronic fatigue, dehydration, bone weakness, muscle weakness, loose teeth, impotency, premature ejaculation, debility, constipation, senility, rheumatism, nervous exhaustion, memory loss, drug withdrawal symptoms, anxiety and arthritis pain in the knee. Extracts from W somnifera inhibit transcription factor nuclear factor kappa B $(\mathrm{NF}-\mathrm{kB})$; thus, it acts as an anti-inflammatory agent. This has been attributed to its ability to interact with IKKB, a kinase that is responsible for the nuclear translocation of NF- $\mathrm{kB}$ and activation of inflammatory signalling pathways (37).

\section{Pausinystalia johimbe (formerly Corynanthe johimbe)}

Yohimbine is the principal alkaloid of the bark of the West African evergreen $P$ johimbe (formerly known as $C$ johimbe), family Rubiaceae. The main active chemical present in $P$ johimbe bark is yohimbine hydrochloride (an indole alkaloid), which has stimulant and aphrodisiac effects. However, the levels of yohimbine that are present in $P$ johimbe bark extract are variable and often very low. Therefore, although $P$ johimbe bark has traditionally been used to treat ED (38), there is insufficient scientific evidence to form a definitive conclusion in this area. It is an antagonist of $\alpha_{2}$-receptors and has 
no direct relation to erection. It acts as a sex motivation stimulant. Yohimbine has been used as both an over-the-counter dietary supplement in the form of an herbal extract, and as a prescription medicine in purified form for the treatment of sexual dysfunction. Yohimbine $20 \mathrm{mg}$ or adjusted dose has been found to be effective in the treatment of orgasmic dysfunction. Yohimbine was recently associated as a treatment for type 2 diabetes mellitus in animal and human models carrying polymorphisms of the alpha-2A adrenergic receptor gene (39). The National Institutes of Health states that yohimbine hydrochloride is the standardized form of yohimbine that is available as a prescription medicine in the United States, and has been shown in human studies to be effective in the treatment of male impotence. Yohimbine hydrochloride USP has been used to treat ED. Controlled studies suggest that it is not always an effective treatment for impotence, and evidence of increased sex drive (libido) is anecdotal only. It cannot be excluded that orally administered yohimbine can have a beneficial effect in some patients with ED. The conflicting results available may be attributed to differences in drug design, patient selection and definition of positive response. Yohimbine has been shown to be effective in the reversal of sexual satiety and exhaustion in male rats, and has also been shown to increase the volume of ejaculated semen in dogs, with the effect lasting at least $5 \mathrm{~h}$ after administration. Yohimbine has also been shown to be effective in the treatment of orgasmic dysfunction in men, and has also been used for the treatment of

\section{REFERENCES}

1. Barkin J. Erectile dysfunction and low testosterone: Cause or an effect? Can J Urol 2010;(Suppl 1):2-11.

2. Berookhim MB, Barchama N. Medical implications of erectile dysfunction. Med Clin North Am 2011;95:213-21.

3. Andersson KE. Pharmacology of penile erection. Pharmacol Rev 2001;53:417-50.

4. Standring S. Editor-in-Chief, Gray's Anatomy: The Anatomical Basis of Clinical Practice, 40th edn. Edinburg: Elsevier Churchill Livingstone 2008:1271-4.

5. Corbin JD, Francis SH, Webb DJ. Phosphodiesterase type 5 as a pharmacologic target in erectile dysfunction. Urology 2002:60:4-11.

6. Meldrum DR, Gambmone JC, Morris MA, Ignarro LJ. A multifaceted approach to maximize erectile function and vascular health. Fertil Steril 2010;94:2514-20

7. Napoli C, de Nigris F, Williams-Ignarro S, Pignalosa O, Sica V, Ignarro LJ. Nitric oxide and atherosclerosis. Nitric Oxide 2006;15265-79.

8. Neal MJ. Medical Pharmacology at a Glance, 5th edn. Blackwell Publishing, 2005;21.

9. Srilatha B, Adaikan PG, Moore PK. Possible role for the novel gasotransmitter hydrogen sulphide in erectile dysfunction. A pilot study. Eur J Pharmacology 2006;535:280-2.

10. Carneiro FS, Webb RC, Tostes RC. Emerging role of TNF alpha in erectile dysfunction. J Sex Med 2010;7:3823-34.

11. Yoshimura N, Kato R, Chancellor MB, Nelson JB, Glorioso JC. Gene therapy as future treatment of erectile dysfunction. Expert Opin Biol Ther 2010;10:1305-14.

12. Lysiak JJ, Kavoussi PK, Ellati RT, et al. Angiogenesis therapy for the treatment of erectile dysfunction. J Sex Med 2010;7:2554-63.

13. Andersson KE, Wagner G. Physiology of penile erection. Physiological Rev 1995;75:191-236.

14. Ignarro LJ, Bush PA, Buga GM, Wood KS. Nitric oxide and cyclic GMP formation upon electrical field stimulation cause relaxation of corpus cavernosum smooth muscle. Biochem Biophys Res Commun $1990 ; 170: 843-50$

15. Holmquist $\mathrm{H}$, Hedlund $\mathrm{H}$, Andersson K-E. Characterization of inhibitory neurotransmission in the isolated corpus cavernosum from rabbit and man. J Physiol 1992;449:295-311.

16. Chauhan NS, Sharma V, Dixit VK, Thakur M. A review on plants used for improvement of sexual performance and virility. Biomed Res Int 2014; (Epub ahead of print August 18).

17. Bhat R, Karim AA, Tongkat Ali (Eurycoma longifolia Jack): A review on its ethanobotany and pharmacological importance. Fitoterapia 2010;81:669-792. sexual side effects caused by some antidepressants, and female hyposexual disorder. Yohimbine has significant side effects, such as anxiety reactions. Higher doses of oral yohimbine may create numerous side effects, such as rapid heart rate, high blood pressure, overstimulation, insomnia and/or sleeplessness. More serious adverse effects may include seizures and renal failure. Yohimbine should not be consumed by individuals with liver, kidney or heart disease, or psychological disorders. The therapeutic index of yohimbine is low; the range between an effective dose and a dangerous dose is very narrow. Side effects include gastrointestinal upset, increased blood pressure, headache, agitation, rash, tachycardia and frequent urination (40).

\section{SUMMARY AND CONCLUSIONS}

Historically, it has been shown that herbal medicines may cure or prevent certain ailments. However, there are very little recorded data available to support the dose, efficacy, side effects and interactions. Because the safety and efficacy of herbal remedies have not been assessed, unlike synthetic drugs, well-controlled and randomized studies are warranted to establish the therapeutic efficy and safety of such products. Determination of side effects and interactions with prescription medicines are also needed. The amount of active ingredients in herbals may vary among preparations; thus, standardization of herbal medicines is required.

18. Kuo PC, Shi LS, Damu AG, et al. Cytotoxic and antimalarial betacarboline alkaloids from the roots of Eurycoma longifolia. J Nat Prod 2003;66:1324-27.

19. Tambi MI, Imran MK. Eurycoma longifolia Jack in managing idiopathic male infertility. Asian J Androl 2010;12:376-80.

20. Farouk AE, Benafri A, Antibacterial activity of Eurycoma longifolia Jack. A Malaysian medicinal plant. Saudi Med J 2007;28:1422-4.

21. Tada H, Yasuda F, Otani K, Doteuchi M, Ishihara Y, Shiro M "Nouveaux quassinoïdes antiulcéreux à partir d'Eurycoma longifolia". Eur J Medicinal Chem 1991;26:345.

22. Ang HH, Ngai TH, Tan TH. Effects of Eurycoma longifolia Jack on sexual qualities in middle aged male rats. Phytomedicine 2003;10:590-3

23. Fernández-Guasti A, Rodríguez-Manzo G. Pharmacological and physiological aspects of sexual exhaustion in male rats. Scand J Psychol 2003;44:257-63.

24. Chen KL, Low BS, Teh CH, Das PK. The effect of Eurycoma longifolia on sperm quality of male rats. Nat Prod Commun 2009;4:1331-6.

25. Zanoli P, Zavatti M, Montanari C, Baraldi M. Influence of Eurycoma longifolia on the copulatory activity of sexually sluggish and impotent male rats. J Ethnopharmacol 2009;126:308-13.

26. Frydrychova S, Opletal L, Macakora K, et al. Effects of herbal preparation on libido and semen quality in boars. Reprod Domestic Anim 2010;46:573-8.

27. Wahab NA, Mokhtar NM, Halim WN, Das S. The effect of Eurycoma longifolia Jack on spermatogenesis in estrogen-treated rats. Clinics (Sao Paulo) 2010;65:93-8.

28. Ang HH, Cheang HS, Yusof AP, Effects of Eurycoma longifolia Jack (Tongkat Ali) on the initiation of sexual performance of inexperienced castrated male rats. Exp Anim 2000;49:35-8.

29. Zakaria Y, Rahmat A, Pihie AH, et al. Eurycomanone induces apoptosis in Hep G2 cells via up-regulation of p53. Cancer Cell Int 2009;9:16.

30. Shuid AN, El-Arabi Z, Effendy NM, et al. Eurycoma longifolia up-regulates osteoprotegerin gene expression in androgen-deficient osteoporosis rat model. BMC Complement Altern Med 2012;12:152.

31. Visavadiya NP, Narasimhacharya AV. Ameliorative effect of Chlorophytum borivilianum root on lipid metabolism in hyperlipaemic rats. Clin Exp Pharm Physiol 2007;34:244-9.

32. Kenjale R, Shah R Sathaye S. Effects of Chlorophytum borivilianum on sexual behaviour and sperm count in male rats. Phyther Res 2008;22:796-801.

33. Giribabu N, Kumar KE, Rekha SS, et al. Chlorophytum boriviliannum (Safed Musli) root extract prevents impairment in characteristics 
and elevation of oxidative stress in sperms of streptozotocin-induced adult male diabetes Wistar rats. BMC Complement Altern Med 2014;14:291.

34. Thakur GS, Bag M, Sanodiya BS, et al. Chlorophytum borivilianum: A white gold for biopharmaceuticals and neutraceuticals. Curr Pharm Biotechnol 2009;10:650-66.

35. Vyawahare NS, Kagathara VG, Kshirsagar AD, et al. Effect of hydroalcoholic extract of Chlorophytum borivilianum tubers in alleviating the diabetic impotency in streptozotocin induced male diabetic rats. Phoog Res 2009;1:314-319.

36. Mishra LC, Singh BB, Dagenais S. Scientific basis for the therapeutic use of Withania somnifera (ashwagandha): A review. Altern Med Review 2000;5:334-346.
37. Heyninck K, Lahtela-Kakkonen M, Van der Veken P, et al. Withaferin A inhibits NFкB activation by targeting cysteine 179 in IKKB. Biochem Pharmacol 2014;91:501-9.

38. Adeniyi AA, Brindley GS, Pryor JP, Ralph DJ. Yohimbine in the treatment of orgasmic dysfunction. Asian J Androl 2007;9:403-7.

39. Rosengren AH, Jokubka R, Tojjar D, et al. Overexpression of alpha2a-adrenergic receptors contributes to type 2 diabetes. Science 2009;327:217-20.

40. Myers A, Barrueto F Jr. Refractory priapism associated with ingestion of yohimbe extract. J Med Toxicol 2009;5:223-5. 